\title{
SCALAR RELATIVISTIC SPIN POLARIZED ELECTRONIC STRUCTURE OF FERROMAGNETIC FCC $\mathrm{GdAl}_{2}$
}

\author{
W. BORGIEL \\ Department of Theoretical Physics, Silesian University \\ Uniwersytecka 4, 40-007 Katowice, Poland \\ AND J. DENISZCZYK \\ Institute of Physics and Chemistry of Metals, Silesian University \\ Bankowa 12, 40-007 Katowice, Poland \\ (Received April 2, 1993; revised version June 3, 1993) \\ One-particle electronic states of ferromagnetic single crystal Laves phase \\ $\mathrm{GdAl}_{2}$ is calculated with the help of augmented spherical waves, based on \\ density functional theory. Density of states, partial densities of states are \\ presented. The behavior of the spectrum in the vicinity of the Fermi energy \\ and the number of occupied states are discussed in detail.
}

PACS numbers: 71.25.Pi, 75.30.-m, 75.50.Cc

\section{Introduction}

Typical magnetic Laves phase structure substances as the $\mathrm{GdAl}_{2}$ [1] and the other $\mathrm{RM}_{2}[2,3](\mathrm{R}$ - rare earth, $\mathrm{M}$ - metal) have been the subject of the extensive experimental as well as theoretical studies [4-7].

Monocrystalline $\mathrm{GdAl}_{2}$ below $171 \mathrm{~K}$ orders ferromagnetically and shows resistivity about $100 \mu \Omega \mathrm{cm}$ [3]. A rather high $\left(7.2 \mu_{\mathrm{B}}\right)$ saturation magnetic moment per Gd atom and sufficiently strong mobility of conducting electrons causes that this substance is a prototype material for testing applicability of whole' $s-f, s-d$ $[8,9]$ family of models. With the help of those models, applying the simplest approximations it was easy to get satisfactory quantitative description of the static magnetic properties, conductivity, ... for finite temperature.

The great progress has been made during the last decade, in the field of using local spin density functional theory (LSDFT)for the description of even struc- 
turally complicated compounds and alloys [10]. Several groups [5, 7, 11, 12] have calculated the one-particle electronic structure of a whole series of compounds and alloys. The method often used and sufficiently efficient was linear muffin tin orbitals (LMTO) method, originated by O.K. Andersen et al. [13], H.L. Skriver [14].

In this paper we report one-particle electronic structure properties of the $\mathrm{GdAl}_{2}$ calculated with the help of the scalar relativistic augmented spherical waves (ASW) method, originally introduced in Ref. [15]. Detailed comparison between ASW and LMTO method is given in Ref. [14]. In many respects ASW is equivalent to LMTO but is rather less efficient numerically and applicable only for structurally ordered materials. The presented calculations were performed on supercomputers.

The paper is organized as follows. In Sec. 2 we summarize the method of calculations paying special care to the compounds aspects. Section 3 contains the description of results for electronic one-particle spectrum, densities of states (DOS), partial densities of states (PDOS). In Sec. 4 we summarize the most important results and also some conclusions are given.

\section{Band-structure calculations}

Band-structure calculations we performed for $\mathrm{GdAl}_{2}$ Laves phase are based on the LSDFT [16] - an approximate version of the exact density functional theory (DFT) of Hohenberg and Kohn [17], Kohn and Sham [18].

Within LSDFT the charge $n(r)$ and magnetization $m(r)$ densities, the partial densities of states and other characteristics of the compounds are obtained by self-consistent solving of the one-particle Kohn-Sham equation (in atomic units)

$$
\left(-\nabla^{2}+V_{\sigma}(r)\right) \Psi_{i, \sigma}(r)=E_{i, \sigma} \Psi_{i, \sigma}(r)
$$

for a single electron with spin $\sigma(\uparrow$ or $\downarrow$ ) in a local periodic potential which is given by

$$
V_{\sigma}(\boldsymbol{r})=V_{\mathrm{ext}}(\boldsymbol{r})+\int \mathrm{d} \boldsymbol{r}^{\prime} \frac{n\left(\boldsymbol{r}^{\prime}\right)}{\left|\boldsymbol{r}-\boldsymbol{r}^{\prime}\right|}+V_{\mathrm{XC}}\left(n(\boldsymbol{r}), n_{\sigma}(\boldsymbol{r})\right) .
$$

$V_{\text {ext }}(r)$ describes the interaction of electrons with the external field which includes the electrostatic interaction with the fixed nuclei. The second term in Eq. (2) represents the Hartree part of electron-electron Coulomb interaction. The exchange-correlation potential $V_{\mathrm{XC}}\left(n(r), n_{\sigma}(r)\right)$ depends on the spin $\sigma$ and within LSDFT is a functional of local charge and spin densities.

The charge density for electrons with spin $\sigma$ is given by

$$
n_{\sigma}(r)=\sum_{i=1}^{N_{\sigma}}\left|\Psi_{i, \sigma}(r)\right|^{2},
$$

where $N_{\sigma}$ is the number of eigenstates with spin $\sigma$ and eigenvalues $E_{i \sigma} \leq E_{\mathrm{F}}$, and $E_{\mathrm{F}}$ is the Fermi energy. The charge $n(r)$ and magnetization $m(r)$ densities are defined as

$$
\begin{aligned}
& n(\boldsymbol{r})=n_{\uparrow}(r)+n_{\downarrow}(r), \\
& m(r)=n_{\uparrow}(r)-n_{\downarrow}(r) .
\end{aligned}
$$


The calculations were performed with the use of the approximate form of $V_{\mathrm{XC}}\left(n(r), n_{\sigma}(r)\right)$ functional given by Morruzi et al. [19]:

$$
\begin{aligned}
& V_{\mathrm{XC}}\left(n(r), n_{\sigma}(r)\right)=-\frac{a}{r_{S}}\left(n_{\sigma}^{\prime}\right)^{1 / 3}+c^{P} \ln \left(1+\frac{r^{P}}{r_{S}}\right) \\
& +\left[c^{F} G\left(\frac{r_{S}}{r^{F}}\right)-c^{P} G\left(\frac{r_{S}}{r^{P}}\right)\right]\left[b\left(\left(2 n_{\sigma}^{\prime}\right)^{1 / 3}-1\right)-\frac{4}{3} f\left(n_{\sigma}^{\prime}\right)\right] \\
& +\left[c^{F} \ln \left(1+\frac{r^{F}}{r_{S}}\right)-c^{P} \ln \left(1+\frac{r^{P}}{r_{S}}\right)\right] f\left(n_{\sigma}^{\prime}\right),
\end{aligned}
$$

with the functions $G(x)$ and $f(x)$ defined as follows:

$$
\begin{aligned}
& G(x)=\left(1+x^{3}\right) \ln \left(1+\frac{1}{x}\right)+\frac{x}{2}-x^{2}-\frac{1}{3}, \\
& f(x)=\left(x^{4 / 3}+(1-x)^{4 / 3}-2^{-1 / 3}\right) /\left(1-2^{-1 / 3}\right) .
\end{aligned}
$$

The variable $r_{S}$ is related to the electron density $n$ by the equation $r_{S}^{3}=3 / 4 \pi n$, $n_{\sigma}^{\prime}=n_{\sigma} / n$ and the numerical parameters take the values $a=1.539, b=5.1298$, $c^{P}=-0.045, c^{F}=-0.0225, r^{P}=21.0, r^{F}=52.9167$.

The band structure calculations based on DFT are $a b$ initio in the sense that the only input parameters are the crystal structure, the lattice parameters and the atomic numbers.

The self-consistent procedure proceeds as follows. First, one assumes certain charge and spin densities (some atomic average), from which the potential (2) is constructed. Then one solves the Kohn-Sham (K-S) equation (1) for the both spin directions and for a dense mesh of $k$-points in the irreducible part of the Brillouin zone. From the $N\left(=N_{\uparrow}+N_{\downarrow}\right)$ states with the lowest eigenenergies $E_{i \sigma}(k)$ the new charge and spin densities are calculated from Eqs. (3) and (4). The procedure is iterated until self-consistency is obtained.

Different methods are involved in order to solve the $\mathrm{K}-\mathrm{S}$ equation, e.g. LCAO, LMTO, LAPW (linear augmented plane waves). They differ in a way of constructing of basis functions in which the eigenfunctions $\Psi_{i, \sigma}(\boldsymbol{r})$ of Eq. (1) are expanded. Our programs use the augmented spherical waves (ASW) method formulated by Williams et al. [15]. The ASW method is an efficient computational scheme to calculate self-consistently electronic structures and ground state properties of periodic crystalline solids. It employs the atomic sphere approximation (ASA) which replaces the Wigner-Seitz (W-S) polyhedra of the solid by overlapping the Wigner-Seitz spheres centered around the atomic positions with a total volume that equals the volume of the solid. Within ASA the potential in the spheres is taken spherically symmetric and $\mathrm{W}-\mathrm{S}$ spheres are taken as the augmentation spheres. This approximation is most accurate for close-packed metals in which space can be filled with $\mathrm{W}-\mathrm{S}$ spheres and whose overlap can be neglected (the overlap less than 30 per cent).

The parameters which enter the ASA based calculations are the radii of W-S spheres. As opposed to the elemental metal, where the $\mathrm{W}-\mathrm{S}$ sphere radius is given by the volume equivalence $\left(V_{\mathrm{sph} .}^{\mathrm{W}-\mathrm{S}}=V_{\text {cell }}^{\mathrm{W}-\mathrm{S}}\right)$, the radii of W-S spheres centered at different atoms in unit cell of compound cannot be determined uniquely. There is 
no unique way to define volumes for specific atomic sites in a compound, though when Vegard's law [20] holds

$$
V\left(\mathrm{~A}_{c} \mathrm{~B}_{(1-c)}\right) \approx c V(\mathrm{~A})+(1-c) V(\mathrm{~B})
$$

where $V(\mathrm{~A})$ and $V(\mathrm{~B})$ are the elemental volumes, a good choice is $V(\mathrm{~A})$ for the $\mathrm{A}$ atoms and $V(\mathrm{~B})$ for the $\mathrm{B}$ ones [20].

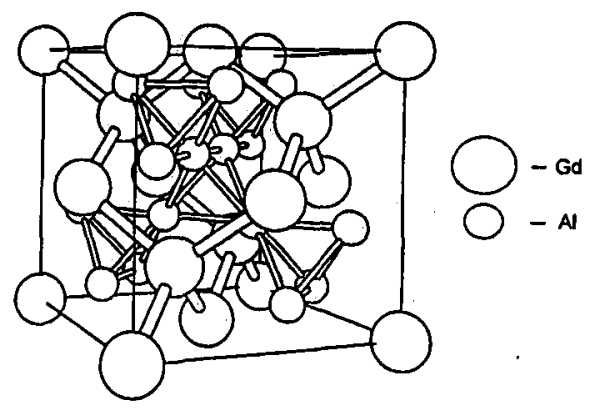

Fig. 1. Sketch of the $\mathrm{GdAl}_{2}$ Laves phase crystallographic structure. Bigger and smaller circles represent $\mathrm{Gd}$ and $\mathrm{Al}$ atoms, respectively.

The crystal structure of $\mathrm{GdAl}_{2}$ in the cubic Laves phase is shown in Fig. 1. The Gd atoms form a diamond sublattice, i.e. two interpenetrating fcc lattices. The Al tetrahedra occupy vacant corners in the Gd sublattice, so that all atoms pack closely. The space group of the cubic Laves phase crystal is $F d 3 m$ and the reciprocal lattice is body centered cubic.

The considered $\mathrm{GdAl}_{2}$ compound does not obey Vegard's law. Some volume contraction accompany the compound formation

$$
V\left(\mathrm{GdAl}_{2}\right)<V(\mathrm{Gd})+2 V(\mathrm{Al})
$$

with $V\left(\mathrm{GdAl}_{2}\right) /(V(\mathrm{Gd})+2 V(\mathrm{Al})) \approx 0.93$. The sizes of $\mathrm{GdAl}_{2}$ compound constituents change relative to their elemental counterparts. These changes are associated with bringing the $\mathrm{W}-\mathrm{S}$ cell boundaries to a common electron density.

To establish the $\mathrm{W}-\mathrm{S}$ spheres' radii for $\mathrm{Gd}$ and $\mathrm{Al}$ atom sites we follow the argumentation of Watson and Bennet [20]. The $\mathrm{AB}_{2}$ Laves structures generally involve larger, lower-valent $A$ atoms which contract upon compound formation and smaller, higher-valent $B$ atoms which expand. If the contraction associated with compound formation is large, the volume decrease in the contracting $\mathrm{A}$ atoms is larger in magnitude than the volume increase in the $\mathrm{B}$ atoms, i.e.

$$
\left|\Delta V_{\mathrm{A}}\right| \gg\left|\Delta V_{\mathrm{B}}\right|
$$

and a good first approximation is to set $\Delta V_{B}$ equal to zero. Providing that the contraction effects are accounted for, the occurrence of the Laves phases is associated with the volume ratio [20]:

$$
\left(\frac{V(\mathrm{~A})}{V(\mathrm{~B})}\right)^{1 / 3}=1.22 \pm 0.14
$$

Following the above arguments we assume that $\Delta V_{\mathrm{Al}}=0$, and the $\mathrm{W}-\mathrm{S}$ sphere radius for $\mathrm{Al}$ atomic site we took equal the $\mathrm{W}-\mathrm{S}$ sphere radius of $\mathrm{Al}$ in 
elemental fcc crystal $R_{\mathrm{Al}}^{W-S} \approx 2.9909$ a.u. Having the $\mathrm{W}-\mathrm{S}$ sphere radius for $\mathrm{Al}$ atomic site, the $\mathrm{Gd} \mathrm{W}-\mathrm{S}$ sphere radius we have determined from the volume equation

$$
n_{\mathrm{Gd}} \frac{4 \pi}{3}\left(R_{\mathrm{Gd}}^{\mathrm{W}-\mathrm{S}}\right)^{3}+n_{\mathrm{Al}} \frac{4 \pi}{3}\left(R_{\mathrm{Al}}^{\mathrm{W}-\mathrm{S}}\right)^{3}=\left(a_{\mathrm{L}}^{0}\right)^{3},
$$

where $n_{\mathrm{Gd}}\left(n_{\mathrm{Al}}\right)$ are the numbers of $\mathrm{Gd}(\mathrm{Al})$ atomic sites in $\mathrm{GdAl}_{2}$ Laves structure unit cell with the lattice constant $a_{L}^{0}$.

Taking into account the experimentally measured lattice constant of $\mathrm{GdAl}_{2}$ Laves phase equal to 14.9282 a.u. [1, 21], the Gd W-S sphere radius from Eq. (9) was found equal to $R_{\mathrm{Gd}}^{\mathrm{W}-\mathrm{S}}=3.5769$ a.u. The volumes of $\mathrm{W}-\mathrm{S}$ spheres centered at $\mathrm{Gd}$ and $\mathrm{Al}$ atomic sites calculated with the above given radii yield

$$
\left(\frac{V^{\mathrm{W}-\mathrm{s}}(\mathrm{Gd})}{V^{\mathrm{W}-\mathrm{S}}(\mathrm{Al})}\right)^{1 / 3} \approx 1.196
$$

which agrees with the value given in Eq. (8).

We set the origin of coordinates at the middle point of the nearest neighbor $\mathrm{Gd}-\mathrm{Gd}$ lines. Thus the positions of the six atoms which form a unit cell are $(0,0,0) a_{\mathrm{L}}^{0}, \quad\left(\frac{1}{4}, \frac{1}{4}, \frac{1}{4}\right) a_{\mathrm{L}}^{0} \quad$ for $\mathrm{Gd}$ atoms and $\left(\frac{1}{8}, \frac{1}{8},-\frac{3}{8}\right) a_{\mathrm{L}}^{0}, \quad\left(-\frac{1}{8}, \frac{1}{8}, \frac{3}{8}\right) a_{\mathrm{L}}^{0}$, $\left(-\frac{1}{8},-\frac{1}{8},-\frac{3}{8}\right) a_{\mathrm{L}}^{0},\left(\frac{1}{8},-\frac{1}{8}, \frac{3}{8}\right) a_{\mathrm{L}}^{0}$ for $\mathrm{Al}$ atoms.

Sticht and Kübler [22] performed local-spin-density functional calculations of ground-state properties of hcp ferromagnetic Gd. They presented two different types of calculations. In the first set of calculations they treated the seven $4 f$ electrons of $\mathrm{Gd}$ as a half-filled core state, aligning all the spins such that they give a magnetic moment of $7 \mu_{\mathrm{B}}$. This assumption led to an unacceptable value of total magnetic moment. They concluded that treating the $4 f$ electrons of $\mathrm{Gd}$ as core states is an unacceptable approximation. In the second set of calculations they treated the $4 f$ electrons as valence states hybridizing with the other valence electrons. The result for the magnetic moment they obtained was in excellent agreement with the experimental value.

In our calculations we also treat the $4 f$ electrons of $\mathrm{Gd}$ atoms as valence states. The starting crystal charge and spin densities are a superposition of self-consistent charge and spin densities for neutral atoms Gd with configuration Xe, $4 f^{7}, 5 d^{0.5}, 6 s^{1}$ for spin up and $\mathrm{Xe}, 4 f^{0}, 5 d^{0.5}, 6 s^{1}$ for spin down. The configuration of neutral $\mathrm{Al}$ atoms were chosen as $\mathrm{Ne}, 3 s^{1}, 3 p^{0.8}$ and $\mathrm{Ne}, 3 s^{1}, 3 p^{0.2}$ for spin up and down, respectively. In the above $\mathrm{Xe}$ and $\mathrm{Ne}$ are the abbreviations of the atomic configurations of xenon and neon, respectively.

\section{Results and discussion}

We performed calculations using scalar relativistic ASW [23] which includes all relativistic effects except spin-orbit coupling. The self-consistency was achieved with $182 k$-mesh points after 16 iterations. In order to obtain a good statistic in DOS, the last iteration was done with $408 k$ points. Additionally, the results from the last converged iteration were linearly splined. In the end, we got relatively smooth curves for all total and partial densities of states. The accuracy of the one-particle spectrum was less than $0.05 \mathrm{eV}$. 
Figure 1 presents crystal structure of $\mathrm{GdAl}_{2}$. The bigger circles correspond to $\mathrm{Gd}$ atoms and the smaller ones to $\mathrm{Al}$ atoms. The hatched areas which form pyramids represent the nearest neighbor sets of $\mathrm{Al}$ atoms. One can easily see that during alloying (i.e. when $\mathrm{Al}$ atom is replaced by another type of atom) we have several nonequivalent space positions.

In Fig. 2 the single particle electronic structure of the ferromagnetic $\mathrm{GdAl}_{2}$ for standard set of $k$-vectors from the first Brillouin zone is shown. The solid lines correspond to the spin up spectrum and the broken ones to the down one. Ex-

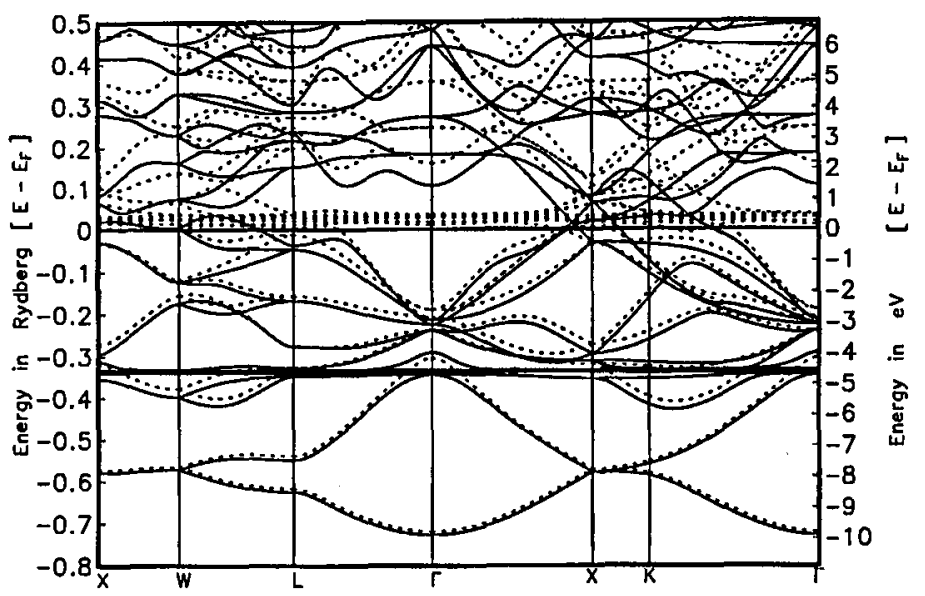

Fig. 2. Ground-state spin up (solid line) and spin down (broken line) band structure of $\mathrm{GdAl}_{2}$ as a function of the wave vector obtained from a scalar-relativistic ASW calculations.

tremely flat dispersion curve $4.5 \mathrm{eV}$ below the Fermi level belongs to $4 f$ multiplet. The width of the $4 f$ down bands is about $0.38 \mathrm{eV}$. In Hund's rule language we can say that all seven $4 f-\uparrow$ electrons are coupled ferromagnetically in the atomic sense and give $7 \mu_{\mathrm{B}}$ contribution to the magnetic moment. The spin down $4 f$ electrons form a broader unoccupied subband located slightly above the Fermi level. The width of the $4 f-\downarrow$ subband is about $0.56 \mathrm{eV}$ (wider than for the $4 f-\uparrow$ states the electrons in states with higher energy have greater mobility). The hybridization with states having another symmetry is rather small. The splitting (binding energy) between $4 f-\uparrow$ and $4 f-\downarrow$ peaks is $5.2 \mathrm{eV}$. This splitting is too small. From inverse photoemission measurement [24] it should be about $12 \mathrm{eV}$. The reason of this striking divergency comes from the approximations used for exchange and correlation potential from Eq. (5).

In Fig. 3 the total single particle density of states is presented (thin vertical line marks the position of the Fermi level). The single particle DOS was defined

$$
\rho_{\sigma}(E)=\frac{1}{N} \sum_{k, m} \delta\left(E-E_{k m \sigma}\right)
$$




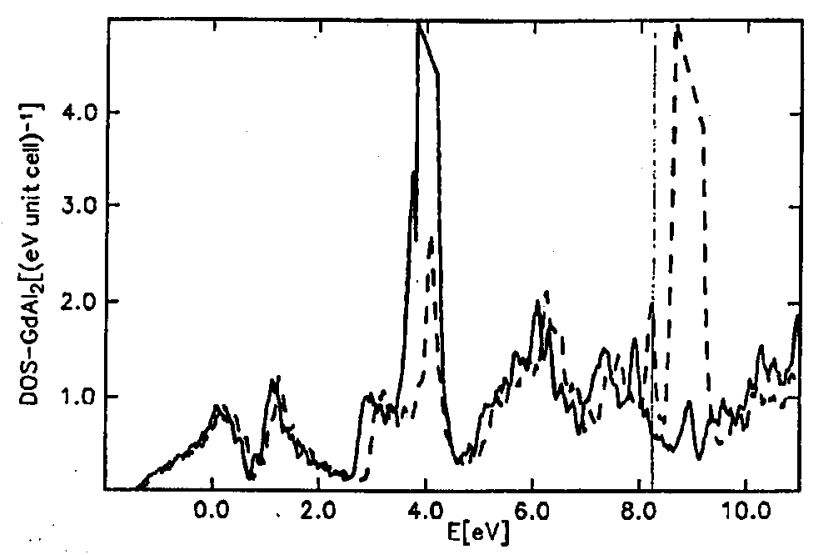

Fig. 3. Total density of states per unit cell for spin up (solid line) and spin down (broken line) as a function of energy $(\mathrm{eV})$ calculated from a scalar-relativistic ASW method.

where $E_{k m \sigma}$ is the quantity obtained from solving the Kohn-Sham equation (1) for solid state and interpreted here as a single particle energy for the electron, directly. The spin up spectrum (solid line) is shifted to the lower energy relatively to the spin down spectrum (broken line). The difference between both spin DOS, which is directly connected with exchange splitting, is strongly energy dependent. The spin down DOS has a sharp maximum exactly at the energy $E=E_{\mathrm{F}}$. The spin up DOS shows also a sharp peak near $E_{\mathrm{F}}$ but shifted to the lower energies. The character of the states near the Fermi energy can be found from the analysis of the projected partial densities of states defined by

$$
\rho_{i \sigma m}(E)=\frac{1}{N} \sum_{\text {occ.st. }} \int\left|\Psi_{i m \sigma}(r)\right|^{2} \mathrm{~d}^{3} r
$$

where $i$ denotes the type of atom ( $\mathrm{Gd}$ or $\mathrm{Al})$ and $m$ enumerates basis function $(m=s, p, d, f)$. Integration is taken over whole Wigner-Seitz sphere around the $i$-atom.

Figures 4 and 5 present PDOS for Gd atom and $\mathrm{Al}$ atom, respectively, projected on the states $s, p, d$. Figure 4 shows that over $80 \%$ of states near Gd atom have $d$ character. From Fig. 5 we see that more than $80 \%$ of states around $\mathrm{Al}$ atom belong to $p$ states. In both cases, PDOS analyzed in the vicinity of $E_{\mathrm{F}}$ shows that states with spin down are dominating. Numerical values for total DOS, PDOS at the Fermi energy and for magnetic moments decomposed into the contributions coming from different states are given in Table I and Table II. 


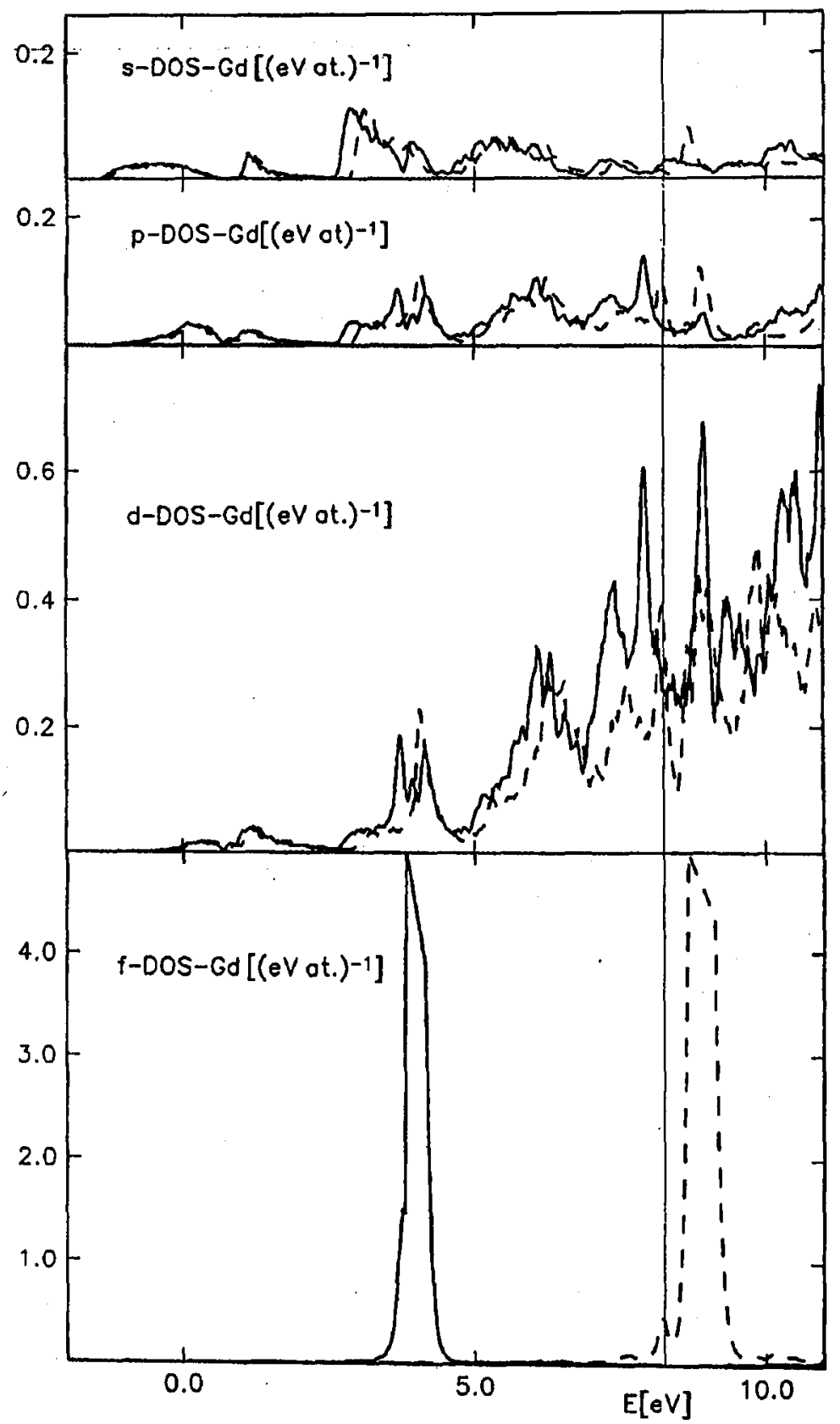

Fig. 4. Partial densities of states per Gd atom for spin up (solid line) and spin down (broken line) as a function of energy (eV) calculated within a scalar-relativistic ASW method and projected on the $s-, p-, d-, f$ basis states. The vertical thin line marks the position of the Fermi level. 
TABLE I

Calculated density of states at the Fermi energy decomposed into the contributions of electron states with different character $l$ and type of atom.

\begin{tabular}{|c|c|c|c|c|}
\hline$l$ & Atom & $\begin{array}{c}\rho_{\uparrow}\left(E_{\mathrm{F}}\right) \\
{[1 / \mathrm{eV} \text { at. }]}\end{array}$ & $\begin{array}{c}\rho_{\downarrow}\left(E_{F}\right) \\
{[1 / \mathrm{eV} \text { at. }]}\end{array}$ & $\begin{array}{l}\rho_{\uparrow+\downarrow}\left(E_{F}\right) \\
{[1 / \mathrm{eV} \text { at. }]}\end{array}$ \\
\hline$s$ & $\mathrm{Gd}$ & 0.024 & 0.010 & 0.034 \\
\hline$p$ & $\mathrm{Gd}$ & 0.026 & 0.088 & 0.114 \\
\hline$d$ & Gd & 0.254 & 0.385 & 0.639 \\
\hline$f$ & Gd & 0.001 & 0.459 & 0.460 \\
\hline \multicolumn{4}{|c|}{$\rho_{\text {total }}^{\mathrm{Gd}}\left(E_{\mathrm{F}}\right)$} & 1.247 \\
\hline$s$ & Al & 0.029 & 0.046 & 0.075 \\
\hline$p$ & $\mathrm{Al}$ & 0.095 & 0.433 & 0.528 \\
\hline$d$ & Al & 0.010 & 0.061 & 0.071 \\
\hline \multicolumn{4}{|c|}{$\rho_{\text {total }}^{\mathrm{Al}}\left(E_{\mathrm{F}}\right)$} & 0.674 \\
\hline \multicolumn{4}{|c|}{$\rho_{\text {total }}^{\mathrm{GdAl}_{2}}\left(E_{\mathrm{F}}\right)[1 / \mathrm{eV}$ f.u. $]$} & 2.595 \\
\hline \multicolumn{4}{|c|}{$\rho_{\text {total }}^{\mathrm{GdAl}_{2}}\left(E_{\mathrm{F}}\right)[1 / \mathrm{eV}$ unit cell $]$} & 5.190 \\
\hline \multicolumn{4}{|c|}{$\rho_{\text {total }}^{\text {exper. }}\left(E_{\mathrm{F}}\right)[1 / \mathrm{eV}$ f.u. $]$} & $2.25 \pm 0.2^{*}$ \\
\hline
\end{tabular}

${ }^{*}$ Reference 3.

TABLE II

Calculated numbers of valence states and magnetic moments decomposed according to different atom type and angular momentum $l$.

\begin{tabular}{|c|c|c|c|c|c|}
\hline$l$ & Atom & $N_{\uparrow}$ & $N_{1}$ & $N_{\uparrow}+N_{\downarrow}$ & $\begin{array}{c}\mu_{l} \\
{\left[\mu_{\mathrm{B}} / \text { at. }\right]}\end{array}$ \\
\hline$s$ & $\mathrm{Gd}$ & 0.256 & 0.230 & 0.486 & 0.026 \\
\hline$p$ & $\mathrm{Gd}$ & 0.333 & 0.281 & 0.614 & 0.052 \\
\hline$d$ & $\mathrm{Gd}$ & 1.004 & 0.711 & 1.715 & 0.293 \\
\hline$f$ & $\mathrm{Gd}$ & 7.000 & 0.218 & 7.218 & 6.782 \\
\hline \multicolumn{5}{|c|}{$\mu_{\text {total }}^{\mathrm{Gd}}$} & 7.153 \\
\hline$s$ & $\mathrm{Al}$ & 0.567 & 0.584 & 1.151 & -0.017 \\
\hline$p$ & $\mathrm{Al}$ & 0.826 & 0.833 & 1.659 & -0.007 \\
\hline$d$ & $\mathrm{Al}$ & 0.085 & 0.081 & 0.166 & 0.004 \\
\hline \multicolumn{4}{|c|}{$\mu_{\text {total }}^{\mathrm{Al}}$} & \multicolumn{2}{|r|}{-0.020} \\
\hline \multicolumn{4}{|c|}{$\mu_{\text {total }}^{\mathrm{GdAl}_{2}}\left[\mu_{\mathrm{B}} /\right.$ f.u. $]$} & \multicolumn{2}{|r|}{7.113} \\
\hline \multicolumn{4}{|c|}{$\mu_{\text {total }}^{\mathrm{GdAl}_{2}}\left[\mu_{\mathrm{B}} /\right.$ unit cell $]$} & \multicolumn{2}{|r|}{14.226} \\
\hline \multicolumn{4}{|c|}{$\mu_{\text {total }}^{\text {exper. }}\left[\mu_{\mathrm{B}} /\right.$ f.u. $]$} & \multicolumn{2}{|r|}{$7.2^{*}$} \\
\hline
\end{tabular}

${ }^{*}$ Reference 25. 


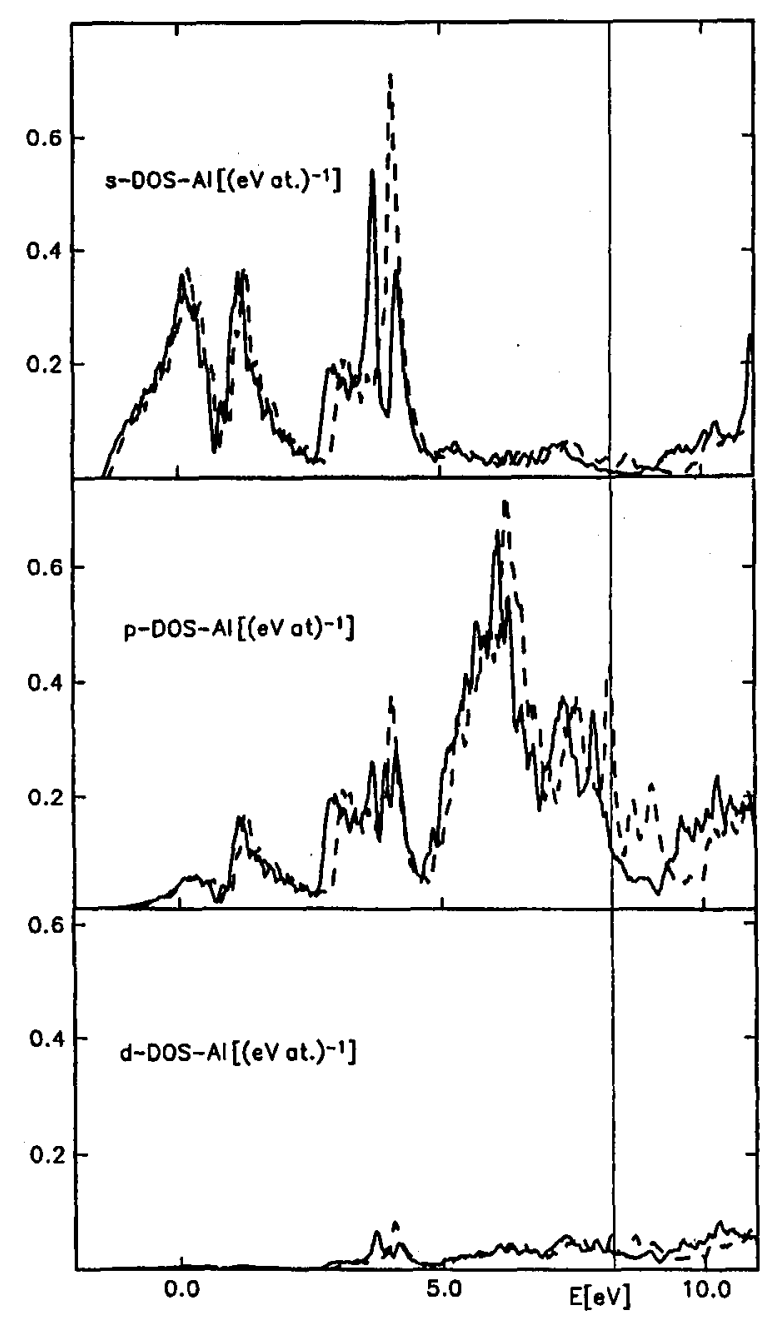

Fig. 5. Partial densities of states per $\mathrm{Al}$ atom for spin up (solid line) and spin down (broken line) as a function of energy (eV) calculated within a scalar-relativistic ASW method and projected on the $s-, p-, d$ basis states. The vertical thin line marks the position of the Fermi level.

\section{Remarks and conclusions}

We have presented one-particle ground state structure of the ferromagnetic $\mathrm{GdAl}_{2}$. The spin up (solid line) and spin down (broken line) band structure are shown in Fig. 2. The total DOS per unit cell is shown in Fig. 3. The spin up $4 f$ states form a narrow peak located $5.2 \mathrm{eV}$ below the Fermi level similarly as in hep Gd [22]. The spin down $4 f$ bands are broader (greater mobility of the electrons 
in states with higher energy) and are located just above the Fermi level. The calculated total magnetic moment per formula unit was $7.113 \mu_{\mathrm{B}}$. Experimental value for saturation magnetic moment was measured to be equal to $7.2 \mu_{\mathrm{B}}$ [25].

Hund's rule for $4 f$ up electrons is perfectly reproduced. We have seven $4 f-\uparrow$ electrons coupled ferromagnetically which in effect give simulation of localized magnetic moment with magnitude $7 \mu_{\mathrm{B}}$. We have found a small contribution to the $4 f$ magnetization coming from $4 f-\downarrow$ states. The magnetic $4 f$ moment from $G d$ atom was evaluated as $6.782 \mu_{\mathrm{B}}$. Nearly the same value for that moment $\left(6.86 \mu_{\mathrm{B}}\right)$ was calculated by Sticht and Kübler [22] for ferromagnetic hcp Gd (they have applied the same method of calculations - ASW). In conclusion we can say that in many global aspect, one-particle spectra for $\mathrm{Gd}$ and $\mathrm{GdAl}_{2}$ are similar.

The calculated net magnetic moment $7.113 \mu_{\mathrm{B}}$ indicates a conduction electron magnetization of $0.331 \mu_{\mathrm{B}}$. The magnetic moment which comes from $\mathrm{Al}$ atoms was established as very small and negative. The negative sign suggests weak antiferromagnetic coupling between magnetic moments of $\mathrm{Gd}$ and $\mathrm{Al}$ and is consistent with RKKY picture [26]. The details were given in Table I and II.

Total density of states on the Fermi level was evaluated as 62.67 [1/Ryd. unit cell]. From this value we can evaluate approximately electronic contribution to the specific heat

$$
C_{\mathrm{e}}=\gamma T, \quad \gamma=2 \eta^{2} / 3 k_{\mathrm{B}}^{2} \rho\left(E_{\mathrm{F}}\right) .
$$

The obtained numerical value for $\gamma$ was too high. The reason was mainly due to the relatively high contribution from narrow $4 f-\downarrow$ states (see Fig. 3).

By analyzing the partial densities of states given in Fig. 4 and 5 we found that $d$ states of $\mathrm{Gd}$, and $p$ states of $\mathrm{Al}$ atoms are dominating in the vicinity of the Fermi level. Especially large contribution comes from $3 d-\downarrow$ of $\mathrm{Gd}$ and $3 p-\downarrow$ of $\mathrm{Al}$ states and this contribution is more than twice larger than that from the other states. The binding energy for the $4 f$ shell was found to be $5.2 \mathrm{eV}$ (see Fig. 4) and is too small compared with the photoemission data [24]. Too small binding energy and in effect relatively high nonphysical contribution to the total density of states coming from $4 f-\downarrow$ states is the consequence of the highly nonhomogeneous $4 f$ electron density and modeling nature of LSDFT.

In the literature one can find constructions which allow to improve the results of the presented above band structure calculations. One possible way is to develop many particle models in such a manner that the results which they yield for the ground state cover in many respects with those obtained within LSDFT formalism. In general, within such a recipe it is not possible to define uniquely parameters of many particle models. Such successful trials of improving of the ground state LSDFT calculations for insulators (EuO, EuS) and metal (Ni) were presented in Refs. [9] and [27], respectively.

There is also a different method of improving the LSDFT based band structure calculations. It was established [28] that the molecular field approximation (MFA) applied to the Hubbard Hamiltonian in the $U / W \gg 1$ limit fits surprisingly well in the case of the half-filled band. In the recent accurate spin polarized DFT calculations for insulating magnetic materials, Anisimov et al. [29] built into the formalism a procedure which treats the half-filled $d$-band electrons more accurately than the homogeneous Stoner-like mean-field approximation. The authors 
proposed modifications of the $V_{\mathrm{XC}} \mathrm{Eq}$. (5) for electrons of strongly correlated half-filled $d$-bands. Modifications are based on multiband Hubbard Hamiltonian treated within the MFA. They applied a special scheme of determination of the Hubbard parameter $U$ and exchange constant $J$. The obtained results are more accurate than LSDFT ones especially when compared with spectroscopic experimental data. Energy positions for narrow $3 d$ down spin states and satellite spectrum is in good agreement with measurements.

The method proposed in [29] is also applicable to $f$ electrons in the metallic $\mathrm{GdAl}_{2}$ compound where the $f$ electrons form strongly correlated spin polarized half-filled bands. Application of the method should improve the results on electronic specific heat and one-particle $4 f$ down spin electronic spectrum. Especially the $4 f$ binding energy should enlarge. The calculations within this approach for $\mathrm{GdAl}_{2}$ are in preparation.

The detailed analysis of the one-particle structure of the whole sequence of the Laves phase compounds with the use of LMTO method is the subject of the current studies performed by Borstel and co-workers [7].

\section{Acknowledgments}

We (W.B. and J.D.) thank Professor Dr. G. Borstel and members of his Group for the hospitality and help during our stay in Osnabrück in 1991. We are also grateful to Prof. G. Borstel for providing us with unpublished materials. Financial support of the Deutsche Sonderforschungsbereich Project SFB 225 is gratefully acknowledged. The work is partially supported by the grant no. 1770/2/91 of the Committee for Scientific Research.

\section{References}

[1] K.H. Bushow, Rep. Prog. Phys. 42, 1373 (1979).

[2] A. Chełkowski, G. Wnętrzak, J. Less-Common Metals 111, 157 (1985).

[3] A. Ślebarski, J. Magn. Magn. Mater. 66, 107 (1987).

[4] A.C. Switendick, Proc. 10th Conf. on Rare Earth Research, Carefree (USA) 1973, Vol. 1, p. 235.

[5] A. Hasegawa, A. Yanase, J. Phys. F, Metal Phys. 10, 847, 2207 (1980).

[6] R. Coehoorn, J. Magn. Magn. Mater. 99, 55 (1991).

[7] M. Magnitskaya, G. Chełkowska, G. Borstel, M. Neumann, H. Ufer, Phys. Rev. B, to be published.

[8] P. Wachter, in: Handbook on the Physics and Chemistry of Rare Earths, Vol. 2, Eds. K.A. Gschneider, L. Eyring, North-Holland, Amsterdam 1979, p. 507.

[9] W. Nolting, W. Borgiel, G. Borstel, Phys. Rev. 37, 7663 (1988).

[10] J. Kudrnovsky, V. Drchal, Phys. Rev. B 41, 7515 (1990).

[11] A. Jezierski, Acta Phys. Pol. A 82, 983 (1992).

[12] M.S.S. Brooks, L. Nordström, B. Johansson, J. Phys. C, Condens. Matter 3, 2357 (1991). 
[13] O.K. Andersen, O. Jepsen, D. Glötzel, Highlights of Condensed-Matter Theory, LXXXIX Corso, Soc. Italiana di Fisica, Bologna 1985, p. 59.

[14] H.L. Skriver, The LMTO Method, Springer, Berlin 1984.

[15] A.R. Williams, J. Kübler, C.D. Gelatt Jr., Phys. Rev. B 19, 6094 (1979).

[16] U. von Barth, L. Hedin, J. Phys. C 5, 1629 (1972).

[17] P. Hohenberg, W. Kohn, Phys. Rev. B 136, 864 (1964).

[18] W. Kohn, L.J. Sham, Phys. Rev. 140, A1133 (1965).

[19] V.L. Moruzzi, J.F. Janak, A.R. Williams, in: Calculated Electronic Properties of Metals, Pergamon, New York 1978.

[20] R.E. Watson, L.H. Bennet, Acta Metall. 32, 491 (1984).

[21] P. Miles, M.A.A. Issa, K.N.R. Taylor, G.J. Bowden, J. Phys. F, Metal Phys. 7, 2421 (1977).

[22] J. Sticht, J. Kübler, Solid State Commun. 53, 529 (1985).

[23] D.D. Koelling, B.N. Harmon, J. Phys. C, Condens. Matter C 10, 3107 (1977).

[24] F.R.Mc Feely, S.P. Kowalczyk, L. Ley, D.A. Shirley, Phys. Lett. A 45, 227 (1973).

[25] E.W. Lee, J.F.D. Montenegro, J. Magn. Magn. Mater. 22, 282 (1981).

[26] J.M. Ziman, Principles of the Theory of Solids, Cambridge University Press, London 1972.

[27] W. Borgiel, W. Nolting, Z. Phys. B 78, 241 (1990).

[28] B. Brandow, Adv. Phys. 26, 651 (1977).

[29] V.I. Anisimov, J. Zaanen, O.K. Andersen, Phys. Rev. B 44, 943 (1991). 